\title{
High Capacitance Nitric Acid-Functionalized MWCNT/PANI composite Supercapacitors under operando conditions.
}

\author{
João C. C. Pregnolato*, Hudson Zanin, Fabio Flaitt, Rafael Vicentini, WIIliam Nunes, Aline Pascom.
}

\section{Abstract}

Nowadays, being able to store and recover energy quickly is becoming increasingly more important for different applications. This Study report on novel electrode material composed of MWCNTs directly synthesized over a fine aluminum foil in CVD then functionalized with Nitric Acid and placed in Coin cells. The electrolyte used was an aqueous solution of Lithium Sulfate 1Mol/L with Sonicator-scattered Pani. This Material showed a good lifetime, solid electric potential stability until $2 \mathrm{~V}$ and improved Capacitance going up to $135 \mathrm{~F} \mathrm{~g}-1$ demonstrating the feasibility of this composite and the techniques involved in near future's State-of-Art supercapacitors.

\section{Key words: \\ Energy, Functionalization, Carbon.}

\section{Introduction}

The study of Carbon-Based Electrochemical Capacitors (ECs) also known as super-capacitors has undergone immense growth over the last years. Much of the approach has been to improve the Energy Storage per mass of these materials since they already exhibit orders of magnitude higher power density than batteries.

In this Context, MWCNT have proved to be useful as an electrode material to work when associated with oxides and polymers because of its stability and because of the synthesis process in CVD.

The objective of this study is to obtain and discuss methods of improving the Capacitance and The electrical potential window of MWCNT

\section{Results and Discussion}

First, the MWCNT is synthesized on thin rectangular aluminum $(6 \mathrm{~cm} \times 5.5 \mathrm{~cm})$ foils associated with Nickel Nitrate by electrodeposition in the CVD using Camphor and $\mathrm{NiNO}_{3}$ as catalysts.

Then, the Al foils with recently grown MWCNTs are subjected to different times $(0.5 \mathrm{~h} ; 1 \mathrm{~h} ; 2 \mathrm{~h} ; 3 \mathrm{~h}$ and $6 \mathrm{~h})$ of Nitric Acid (65\%) treatment.

This step is essential for obtaining a larger electric potential window, and the best results were obtained in $3 \mathrm{~h}$.

Thereupon, since Polyaniline is a material well known for its pseudo capacitive reaction in a weakly acid media, it was scattered by Sonicator and placed over the substrate.

After this process, the Capacitance of the cell suffered a small increase and the electric potential remained the same

Chart 1. EDS measurement of the electrode material showing the Mass Ratio of Carbon and Oxigen

\begin{tabular}{|l|l|l|}
\hline $\begin{array}{l}\text { Chemical } \\
\text { Element:: }\end{array}$ & $\begin{array}{l}\text { Weight Before the } \\
\text { Acid treatment }\end{array}$ & $\begin{array}{l}\text { Weight After the } \\
\text { Acid treatment }\end{array}$ \\
\hline \multicolumn{3}{|l|}{} \\
\hline C & $43,01 \%$ & $45,44 \%$ \\
\hline $\mathrm{O}$ & $6,13 \%$ & $29,31 \%$ \\
\hline
\end{tabular}

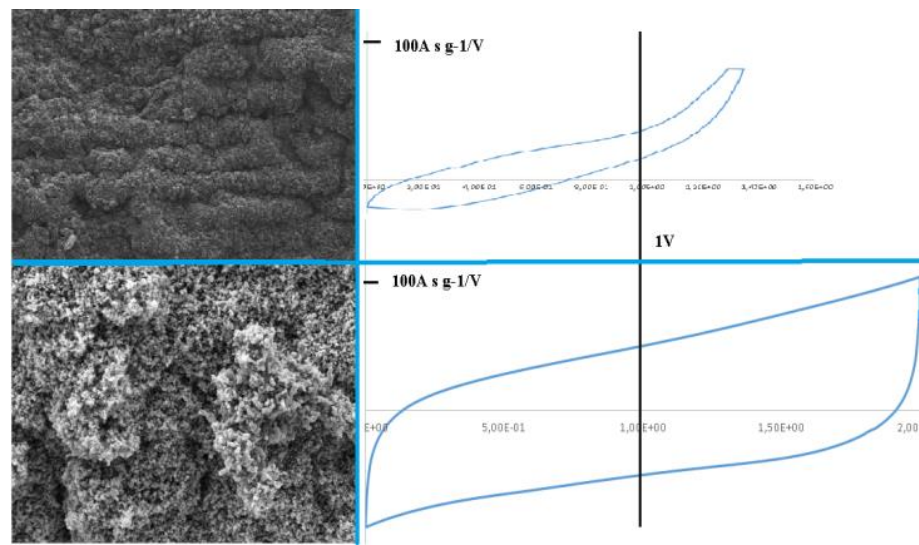

Image 1. Capacitance comparison on the same scale, on top, as-made MWCNT, at bottom with PANI after treatment

In view of the TEM and EDS measurement above, we can conclude that due to the reaction with Acid, we have the formation of oxygenated radicals, that make the material more hydrophilic and also increase the surface area, as a consequence we have an increased capacitance, that we can see in the picture above.

\section{Conclusions}

In order to better understand the results obtained, it is a good approach to split the analysis between $\mathrm{HNO}_{3}$ functionalization and Polyaniline deposition

The functionalization is perceived to be a valuable method of not only increasing the Capacitance but also the Electric Potential Window - which is shown to be the best way of obtaining more favorable energy density (because it has quadratic dependence with V). In this context, the increase in $V$ was solid in $+42,8 \%$.

Moreover, the PANI Deposition showed to improve the final Capacitance of the Electrode.

With this in mind, the best time of Acid Treatment obtained was $2 \mathrm{~h}$ and the best PANI concentration was $0,8 \mathrm{mg}$, achieving $135 \mathrm{~F} \mathrm{~g}-1$, and 2V. Attesting how successful the processes develop are.

\section{Acknowledgement}

The authors are very grateful to LNNano/CNPEM and CCS Nano/FEEC for the technical measurement contribution, to Brazilian funding agency $\mathrm{CNPq}(\mathrm{PIBIC})$ and for Prof. Emerson S. Gonçalves(ITA) for the PANI supply. 